\title{
INSTITUIÇÕES DE LONGA PERMANENCIA PARA IDOSOS: OLHARES SOBRE A PROFISSÃO DO NUTRICIONISTA
}

Fábia Benetti

Alessandra Cardoso Vargas ${ }^{2}$

Marilene Rodrigues Portella ${ }^{3}$

Telma Elita Bertolin ${ }^{4}$

resumo

O envelhecimento populacional está ocorrendo em um contexto de grandes mudanças sociais, culturais, econômicas, institucionais, no sistema de valores e na configuração dos arranjos familiares. A necessidade de cuidado aos mais velhos traz para o cenário a Instituição de Longa Permanência para Idosos (ILPI). Neste contexto, o

1 Nutricionista. Mestranda Bolsista do Programa de Pós-Graduação em Envelhecimento Humano da Universidade de Passo Fundo (UPF). E-mail: fabia_b.14@hotmail.com

2 Mestre em Envelhecimento Humano pela Universidade de Passo Fundo (UPF). Graduada em Educação Física pela Universidade Luterana do Brasil (ULBRA-SM). E-mail: alessandracvargas@hotmail.com

3 Doutora em Enfermagem pela Universidade Federal de Santa Catarina. Docente do Programa de Pós-Graduação em Envelhecimento Humano da Universidade de Passo Fundo (UPF). E-mail: portella@upf.br

4 Doutora em Tecnologia Bioquímica Farmacêutica pela Universidade de São Paulo. Docente do Programa de Pós-Graduação em Envelhecimento Humano da Universidade de Passo Fundo (UPF).

E-mail: telmabertolin@hotmail.com 
nutricionista desempenha papel fundamental na organização dos serviços de nutrição, avaliação e acompanhamento do estado nutricional. Objetivou-se, no presente estudo, conhecer o perfil do profissional de nutrição que trabalha em ILPI e identificar os desafios e perspectivas apontados por eles no contexto da institucionalização. Trata-se de um estudo exploratório/descritivo de natureza qualitativa. Participaram do estudo sete nutricionistas com idade entre 22 e 35 anos. A coleta de dados foi realizada por meio de entrevistas, através de aplicação de questionário semiestruturado sobre o perfil do profissional, dados sociodemográficos, com questões sobre a organização do serviço de nutrição, desafios e perspectivas em relação ao trabalho do nutricionista. Os desafios apontados dizem respeito aos hábitos alimentares dos idosos e as necessidades de modificações; ao descompasso entre prescrição do nutricionista e entendimento dos funcionários sobre as necessidades dos idosos; e ao trabalho com o cardápio condicionado aos recursos oriundos de doações. As perspectivas são otimistas em relação à inserção do profissional neste cenário; necessidade de rigor na fiscalização e luta por remuneração justa e, ainda, investimento na formação acadêmica.

palavras-chave

Institucionalização. Envelhecimento. Idoso. Nutricionista.

\section{Introdução}

O envelhecimento demográfico é um processo irreversível e atinge grande parte da população mundial. A dinâmica da população brasileira, assim como a dos demais países da América Latina, experimenta grandes transformações, expressas, principalmente, pela redução do seu ritmo de crescimento e por mudanças significativas na distribuição etária, resultando no célere aumento do segmento idoso nas próximas décadas (CAMARANO; MELLO; KANSO, 2009).

O avanço no campo das ciências da saúde contribui decisivamente para a longevidade, todavia na idade avançada aumentam as chances de agravamento das doenças crônicas e incapacitantes. Uma das consequências da transição demográfica e epidemiológica é a sobrevida dos idosos na condição de dependência de cuidado (CREUTZBERG; SANTOS, 2003). 
O envelhecimento da população é acompanhado pelo envelhecimento do indivíduo de outros segmentos populacionais, como a População Economicamente Ativa e as famílias. Esse processo altera a vida do indivíduo, as estruturas familiares e a sociedade (CAMARANO; KANSO; MELLO, 2004).

Segundo Camarano e Pasinato (2004), embora em nosso país a institucionalização de idosos não constitua uma prática constante, ela ocorre, em especial, quando a família já não pode dispensar os cuidados necessários a um idoso dependente. Culturalmente, as famílias postergam até o seu limite de cuidado a decisão de colocar o seu idoso em uma instituição de longa permanência para idosos (ILPI).

Sendo assim, a necessidade de cuidados ao idoso por período prolongado e a institucionalização ainda são temas delicados a serem abordados nas famílias e sociedade de modo geral. As causas da institucionalização estão relacionadas à condição de vulnerabilidade da pessoa idosa, à insuficiência de suporte familiar e, em muitos casos, às capacidades física, cognitiva e mental alteradas (CAMARANO; MELLO, 2010).

As ILPIs proporcionam serviços nas áreas sociais, humanas e de saúde, integrando-se ao conjunto dos sistemas societais que abrigam idosos dependentes ou semidependentes e sem família. Essas instituições abrigam idosos em diferentes circunstâncias de vida e saúde, por isso a função que essa instituição desempenha deve ir além de uma residência coletiva. A ILPI deve oferecer serviço de assistência multidimensional gerontológica (CREUTZBERG et al., 2007).

De acordo com a RDC n. 283/2005, a instituição de longa permanência para idosos é destinada à moradia coletiva de pessoas com idade igual ou superior a 60 anos, com ou sem suporte familiar, seja de caráter governamental ou não, constituindo parte da rede de assistencial social. Nestas instituições, deve haver profissionais capacitados para atender esta população, dentre eles, o profissional de nutrição, que deverá realizar atendimentos por 4 horas semanais (BRASIL, 2005a).

A ciência da nutrição teve, nos últimos anos, um expressivo desenvolvimento, que pode ser atestado na expansão dos grupos de pesquisa, publicação de trabalhos e número de pesquisadores. Embora se disponha de conhecimentos técnicos para solucionar a maior parte dos problemas de nutrição existentes, a atuação do nutricionista em algumas áreas, onde a presença dos profissionais é exigida por lei, tem sido ainda hoje tímida e incipiente (BOOG, 2008).

Diante da crescente demanda da população institucionalizada, se faz necessária a prática do nutricionista dentro das ILPIs, implantando programas alimentares adequados, mediando e promovendo melhor atendimento nas dimensões biológicas, sociais e psicológicas, discutindo todo o envolvimento político que este idoso representa (CREUTZBERG et al., 2007). 
Neste sentido, na presente pesquisa objetivou-se conhecer o perfil do profissional de nutrição que trabalha em ILPIs e identificar os desafios e perspectivas apontados por eles, no contexto da institucionalização.

\section{Metodologia}

O presente estudo foi desenvolvido em conformidade com as instruções contidas na Resolução 196/96 do Conselho Nacional de Saúde do Ministério da Saúde para estudos com seres humanos; ainda, foi submetido à apreciação do Comitê de Ética em Pesquisa da Universidade de Passo Fundo e aprovado conforme o parecer $n^{\circ}$ 393/2011. Todos os sujeitos concordaram em participar da pesquisa formalizada pela assinatura do Termo de Consentimento Livre e Esclarecido.

Trata-se de uma pesquisa exploratório/descritiva de natureza qualitativa, realizada no município de Passo Fundo - RS. Inicialmente procedeu-se o mapeamento dos cadastros das instituições de longa permanência para idosos, obtidos junto ao Conselho Municipal do Idoso, sendo identificadas 18 ILPIs na cidade do estudo; destas, oito aceitaram participar da pesquisa. Foram localizadas apenas sete profissionais de nutrição - e uma dessas profissionais atua em duas instituições -, todas do sexo feminino, com idade entre 22 e 35 anos.

A coleta de dados foi realizada mediante entrevistas, aplicando-se um questionário semiestruturado com perguntas sobre o perfil do profissional, dados sociodemográficos, sobre a organização do serviço, desafios e perspectivas em relação ao trabalho do nutricionista no contexto das ILPIs. Os dados foram sistematizados em diferentes categorias de análise, tomando por referência a análise temática proposta por Minayo (2004).

\section{Resultados e discussões}

Quanto às características sociodemográficas, foram entrevistadas sete profissionais de nutrição, todas do gênero feminino, com idade variando de 22 a 35 anos, e tempo de formação, em média, de seis anos. Conforme os registros das entrevistas, suas experiências nas ILPIs são de dois anos e meio, sem formação gerontológica específica, mas duas destas profissionais possuem especialização em áreas afins.

Não obstante, três entrevistadas salientaram em seus relatos que tiveram a oportunidade de desfrutar apenas de uma disciplina relacionada à área 
gerontológica, mas obtiveram pouco tempo de estágio para realizar a prática com a população em questão.

Outro fato observado neste cenário é o regime de contrato de trabalho, o qual mostra que apenas três profissionais de nutrição são contratadas no regime da Consolidação das Leis Trabalhistas (CLT), o restante trabalha de forma autônoma. De modo geral, possuem uma média de frequência nas ILPIs de seis horas semanais, nas quais desenvolvem atividades referentes à elaboração de cardápios, avaliação nutricional dos idosos institucionalizados, treinamentos para os manipuladores de alimentos. Reconhecem que as horas trabalhadas nas instituições são insuficientes para a realização de um bom trabalho.

Segundo as nutricionistas entrevistadas, os cardápios são modificados a cada 15 dias; as mesmas relatam a necessidade da realização de alterações para os idosos que apresentam patologias específicas, por esse motivo precisam de mais tempo disponibilizado pela instituição para realizar este trabalho. Durante seus relatos, observou-se que seguem as diretrizes da alimentação saudável e o guia alimentar para a população brasileira do Ministério da Saúde, bem como diretrizes específicas conforme a doença apresentada pelos residentes (BRASIL, 2005b).

De acordo com Assis (2005), os hábitos saudáveis incluem alimentação balanceada, prática regular de exercícios físicos, convivência social estimulante, atividade ocupacional prazerosa e mecanismos de atenuação do estresse, dados estes que corroboram com o estudo em questão, uma vez que o profissional de nutrição segue com grande entusiasmo as diretrizes de uma alimentação saudável com os idosos residentes.

A análise dos dados permitiu elaborar duas categorias temáticas que retratam os desafios sob o ponto de vista dos profissionais de nutrição que atuam em ILPIs e as perspectivas relacionadas a seu trabalho como profissional, no contexto da institucionalização.

\subsection{Desafios do ponto de vista dos profissionais de nutrição que atuam nas ILPIS}

No contexto da institucionalização, os profissionais de nutrição se mostram numa situação desafiadora, em que a aceitação do cardápio por parte dos idosos e também pelos profissionais que trabalham na cozinha, a doação de alimentos e a preocupação com o idoso se apresentam como a tônica da conjuntura, como se confere nas falas. 
Um dos desafios é a aceitação do cardápio por parte dos idosos, que vêm com hábitos alimentares preestabelecidos durante a vida. Ainda mais os que, por causa das doenças que apresentam, necessitam restringir a ingestão de determinados alimentos. Eles querem comer o que têm vontade e não pensam na saúde. Então é uma briga só (E1).

Evidencia-se na fala de E1 que a aceitação do cardápio por parte dos idosos se faz um presente desafio que enfrentam no dia a dia de sua profissão, reconhecendo a necessidade de um atendimento diferenciado para cada idoso institucionalizado.

Segundo o estudo de Santelle et al. (2007), o estado nutricional de idosos institucionalizados poderá ser melhorado por meio de medidas que promovam a adequada ingestão de alimentos, como observado neste estudo em que as modificações dietéticas na alimentação dos institucionalizados podem ser necessárias devido a mudanças fisiológicas que podem afetar a habilidade para digerir e absorver alimentos, contudo, sempre proporcionando a esta população um alimento nutritivo e saboroso.

Outro dado relevante relatado pelas nutricionistas entrevistadas foi a relação do estado nutricional inadequado com as altas taxas de morbidade e mortalidade. Segundo elas, a presença de problemas de saúde, interferindo na alimentação do idoso, é referida como algo comum nas instituições, indicando um fato que merece ser valorizado pelas equipes de saúde e pelas pessoas responsáveis pela alimentação, as quais muitas vezes não conseguem obter uma boa relação. Como se confere a seguir:

Tem também a função com as cozinheiras, que nem sempre colaboram com as atividades desenvolvidas que propomos, em função também do próprio alimento que tem no momento. É bem complicado, porque não estou aqui todos os dias para fiscalizar se estão, elas fazem as coisas como bem entendem. Elas trabalham com isso há anos e eu sou nova, então elas têm a impressão que não tenho o que ensinar para elas, que já sabem tudo o que precisam saber (E2).

Evidencia-se aqui a preocupação que os profissionais de nutrição enfrentam em relação ao que se refere principalmente à forma de preparo dos alimentos e à monotonia dos cardápios das instituições, pois se sabe que é necessário considerar, no seu planejamento, necessidades nutricionais, condições econômicas da instituição, hábitos alimentares do público-alvo, variedade e harmonia entre os ingredientes e preparações. Observa-se em consonância com este estudo que os dados das entrevistas corroboram com o estudo de Jomori (2006), no 
qual os fatores relacionados aos alimentos estão ligados diretamente a uma apresentação visual adequada, tipo de preparação, variedade do cardápio, sabor e valor nutricional.

As entrevistadas também afirmaram que muitos alimentos utilizados nas ILPIs onde atuam são oriundos de doações, sendo que estes muitas vezes acabam por encontrarem-se muito próximos do prazo de validade, bem como serem manipulados de forma inadequada pelas cozinheiras, aumentando o risco de contaminação. Como podemos observar nas falas a seguir:

Também tem a parte do aproveitamento das doações, cuidados com esses alimentos desde que chegam, durante a manipulação e como são distribuídos aqui na instituição pelos funcionários da cozinha. Quando vem um alimento de doação, geralmente a quantidade é grande do mesmo alimento. Daí tem que aproveitar porque estragam logo, principalmente quando são frutas e verduras. Daí então os idosos reclamam porque é bolo de maçã, maçã de lanche e de sobremesa (E8).

Um desafio bem importante pra nós da nutrição são os problemas relacionados com a elaboração dos cardápios. Em função de como é compreendido pela cozinheira o cardápio prescrito (E3).

Muitas vezes as cozinheiras mudam o cardápio, principalmente quando é a dona da casa, ela quase sempre altera o cardápio para a redução dos custos com a alimentação aqui na instituição (E5).

Verificam-se, nas falas acima, o quanto se faz importante a presença do profissional de nutrição, e os desafios que enfrentam ao se deparar com a prática profissional dentro de uma Instituição de Longa Permanência para Idosos. Cabe ainda salientar que as ILPIs estudadas objetivam proporcionar uma alimentação saudável e adequada do ponto de vista higiênico e sanitário ao comensal, tentando inserir as doações que recebem neste contexto.

O fato de muitas vezes as funcionárias da cozinha não seguirem os cardápios conforme a prescrição da Nutricionista pode estar relacionado a uma falta de diálogo na equipe, bem como pode também estar associado à falta de treinamento das cozinheiras, que precisam compreender que o cardápio foi montado conforme as necessidades nutricionais específicas para os idosos, e que as modificações realizadas podem vir a tornar a alimentação do dia pobre em alguns nutrientes específicos.

De fato, apenas recentemente têm sido implantadas equipes multiprofissionais nas ILPIs que se destinam a atender as necessidades específicas dos idosos institucionalizados e ainda pode-se encontrar insuficiente atendimento gerontológico em muitas destas. Acreditamos que as descrições sobre os desa- 
fios dos profissionais de nutrição e suas relações possibilitaram a interpretação do significado atribuído à sua vivência nestas instituições.

De forma mais ampla, deve-se estimular mais discussões a respeito do perfil do profissional de nutrição e os desafios enfrentados por eles, no contexto gerontológico, considerando a complexidade do cuidado às pessoas com idade avançada.

\subsection{Perspectivas relacionadas ao trabalho}

\section{do nutricionista no contexto da institucionalização}

Nas falas das entrevistadas nota-se a perspectiva de que serão reconhecidas profissionalmente nas instituições de longa permanência para idosos e mostrarão aos órgãos governamentais o quanto são importantes. Reconhecem que a inserção do nutricionista é tão importante quanto a de qualquer outro profissional e percebem que somente com o passar dos anos o profissional será mais valorizado. É o que se confere nas falas:

Vejo a inserção do profissional Nutricionista com otimismo para o futuro, com maior participação do mesmo. Acredito que nós vamos ser mais valorizados no futuro e não seremos contratados somente por obrigação da lei (E1).

Acredito que a valorização do profissional já melhorou muito [...]. As instituições estão se dando conta da importância de estarmos aqui, embora necessite melhor remuneração (E4).

Verifica-se nas falas que se fazem cada vez mais necessários instituições e profissionais de saúde capacitados para cuidar de idosos, proporcionando qualidade de vida aos mesmos. A função do nutricionista é extremamente importante, visto as inúmeras alterações fisiológicas, psicológicas e sociais que afetam o ato da alimentação, as escolhas e hábitos alimentares dos idosos. Entretanto, seu papel não pode se limitar ao cálculo dos nutrientes e à organização do cardápio. Deverá orientar o pessoal da cozinha e da copa sobre o significado afetivo da refeição e da necessidade de zelar também pelos seus aspectos estéticos. Organizar refeições com sugestões e pedidos dos idosos, criar oportunidades ocasionais para permitir a escolha de alguns pratos pode ser um exercício de autonomia extremamente importante para as pessoas dependentes (PEREIRA; CERVATO, 2002).

Em vista disso, Silva e Cunha (2002) relatam a necessidade de preparo dos profissionais para dar conta das novas exigências da sociedade do conhecimento, pois a informação tornou-se fundamental e a competência dos profissio- 
nais tem sido submetida à pressão frente às novas demandas. Assim, segundo Folle et al. (2009), ao ingressar nessa profissão, assumem-se cada vez mais responsabilidades profissionais e muitas vezes o nutricionista sente-se despreparado, como se confere nesta fala:

Uma perspectiva que tenho é quanto às melhorias na normatização do trabalho do nutricionista em instituições. Tinha que ter uma fiscalização maior quanto à exigência da atuação, assim todos nós iríamos nos qualificar mais [...] Também um aumento na quantidade de horas de trabalho (E5).

Verifica-se na fala de $E 5$ o quanto se sentem muitas vezes desvalorizados em sua profissão, fazendo com que não procurem uma melhor qualificação no mercado de trabalho, ou seja, são desmotivados. Contudo, nem todos sentem assim, uma vez que $E 7$ ressalta em sua fala:

Vejo boas perspectivas futuras quanto à valorização profissional, pois nós mesmos começamos a nos valorizar mais e vejo também que estão abrindo portas para a nossa atuação no segmento das pessoas idosas (E7).

Existem evidências de que o consumo alimentar entre idosos institucionalizados é inadequado em diversos aspectos, fator que implica a necessidade de cuidado e atenção especial, seguidos de monitoramento da situação nutricional. A ocorrência da má nutrição em idosos institucionalizados é um evento de prevalência elevada, estando associado ao maior risco de morbimortalidade, bem como à perda de função e desempenho das atividades de vida diária (PASSOS; FERREIRA, 2010).

Neste contexto a atuação do profissional Nutricionista torna-se de fundamental importância, com objetivo de proporcionar ao idoso uma nutrição adequada e completa em macro e micronutrientes, desta forma contribuindo para o bem estar destes, para que vivam com mais saúde e qualidade de vida.

Sabe-se que a alimentação, desde que nutricionalmente adequada, exerce papel fundamental na promoção, manutenção e recuperação da saúde de pessoas idosas, sendo que as mudanças decorrentes do processo de envelhecimento podem ser atenuadas com uma alimentação adequada e balanceada nos aspectos dietéticos e nutritivos, onde o ser humano é agente de sua própria saúde e não mais um expectador.

Em vista disso, a Resolução de Diretoria Colegiada (RDC) n. 283 de setembro de 2005 da Agência Nacional de Vigilância Sanitária (ANVISA), recomenda que toda a Instituição de Longa Permanência para Idosos tenha no corpo de recursos humanos o profissional de nutrição, que deverá atuar na ILPI no mínimo 4 horas semanais (BRASIL, 2005a). 
O trabalho do nutricionista é de fundamental importância compondo a equipe multiprofissional de uma ILPI, pois este tem o compromisso de proporcionar uma alimentação nutritiva e saudável aos idosos, com qualidade e segurança alimentar, contribuindo para que os mesmos mantenham e/ou recuperem a saúde, e que possam assim desfrutar de independência e qualidade de vida. Para que a vida longa, desejada por todos, seja prazerosa e não onerosa.

\section{Considerações finais}

Quanto ao perfil do profissional Nutricionista que atua no município pesquisado, constatou-se o predomínio de mulheres jovens, que atuam neste segmento específico há pouco tempo, sendo que dentre as pesquisadas nenhuma apresenta especialização na área do envelhecimento humano. Cabe também salientar a baixa carga horária destinada às atividades de trabalho destas profissionais nas ILPIs, o que repercute na dificuldade de prestar um serviço adequado às necessidades do idoso e da instituição.

Por outro lado, os avanços conquistados pela categoria ao longo dos anos são evidentes, dentre essas conquistas podemos destacar a sensível ampliação dos campos de atuação profissional. A exigência legal da presença do profissional nutricionista em ILPI abre um novo campo de trabalho, onde se dedica ao cuidado da alimentação dos idosos, entretanto este cenário mostra fragilidades e dificuldades que o nutricionista enfrenta no seu dia a dia.

Os desafios são grandes assim como a esperança de dias melhores, com maior reconhecimento, respeito às leis e incentivos ao trabalhador, para que este possa desenvolver um trabalho adequado que venha a somar em qualidade para a vida dos idosos institucionalizados.

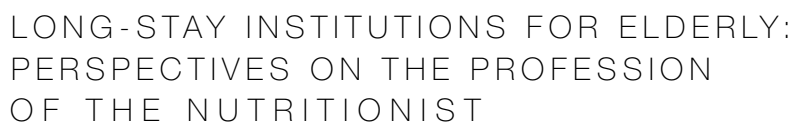

\section{abstract}

Population ageing is occurring in a context of major changes in social, cultural, economic, institutional spheres, in the system of values and in the configuration of family arrangements. The need for care to the elder brings to the fore the Long-Stay Institution for the elderly (ILPI). In this context, the nutritionist has a key role in the nutri- 
tion services organization, evaluation and monitoring of nutritional status. Knowing the profile of the nutritionist professional who works on ILPI and identifying the challenges and perspectives pointed by them in the context of the institutionalization were the aims of the current study. This is a descriptive/exploratory study of qualitative nature. Took part in it seven nutritionists aged from 22 to 35 years. The data were collected through interviews, by the application of semi structured questionnaire on professional profile, sociodemographic data, with questions about the nutrition service organization, challenges and perspectives in relation to the work of the nutritionist. The challenges singled out concern the eating habits of the elderly and the need for modifications; the mismatch between the prescription of the nutritionist and the understanding of staff about the needs of the elderly; and the working with the menu conditioned to resources from donations. The prospects are optimistic in relation to the insertion of the professional in this scenario; need for rigour in supervision and fighting for fair remuneration and, still, investment in the academic training.

keywords

Institutionalization. Aging. Elderly. Nutritionist.

referências

ASSIS, Mônica de. Envelhecimento ativo e promoção da saúde: Reflexão para as ações educativas com idosos. Revista APS, Juiz de Fora, v. 8, n. 1, p. 15-24, jan./ jun. 2005.

BOOG, Maria Cristina Faber. Atuação do nutricionista em saúde pública na promoção da alimentação saudável. Revista Ciência \& Saúde, Porto Alegre, v. 1, n. 1, p. 33-42, jan./ jun. 2008.

BRASIL. Resolução da Diretoria Colegiada RDC 283, de 26 de setembro de 2005. Aprova o Regulamento Técnico que define normas de funcionamento para as Instituições de Longa Permanência para Idosos. Agência Nacional de Vigilância Sanitária. Diário Oficial da República Federativa do Brasil, Brasília, DF, 2005a.

BRASIL. Guia Alimentar para a população brasileira: promovendo a alimentação saudável. Ministério da Saúde - Secretaria de Atenção à Saúde. Coordenação-Geral da Política de Alimentação e Nutrição. Brasília: Ministério da Saúde, 2005b.

CAMARANO, Ana Amélia; KANSO, Solange; MELLO, Juliana Leitão. Como vive o idoso brasileiro? In: CAMARANO, Ana Amélia (Org.). Os novos idosos brasileiros: muito além dos 60? Rio de Janeiro: IPEA, 2004. p. 25-73.

CAMARANO, Ana Amélia; PASINATO, Maria Tereza. Introdução. In: CAMARANO, Ana Amélia (Org.). Os novos idosos brasileiros: muito além dos 60? Rio de Janeiro: IPEA, 2004. p. 1-22. 
CAMARANO, Ana Amélia; MELLO, Juliana Leitão; KANSO, Solange. Juventude e enveIhecimento na Conferência do Cairo: 15 anos depois no Brasil. In: Brasil, 15 anos após a Conferência do Cairo. Campinas: ABEP/UNFPA, 2009. p. 233-290.

CAMARANO, Ana Amélia; MELLO, Juliana Leitão. Cuidados de longa duração no Brasil: o arcabouço legal e as ações governamentais. In: CAMARANO, Ana Amélia (Org.). Cuidados de longa duração para a população idosa: um novo risco social a ser assumido? Rio de Janeiro: IPEA, 2010. p. 68-92.

CREUTZBERG, Marion; SANTOS, Beatriz Regina Lara. Famílias cuidadoras de pessoa idosa: relação com instituições sociais e de saúde. Revista Brasileira de Enfermagem, Brasília, v. 56, n. 6, p. 624-629, nov./dez. 2003.

CREUTZBERG, Marion et al. Long-Term Care Institutions for Elders and the health system. Revista Latino-Americana de Enfermagem, Ribeirão Preto, v. 15, n. 6, p. 1144-1149, Nov./Dec. 2007.

FOLLE, Alexandra et al. Construção da Carreira Docente em Educação Física: Escolhas, Trajetórias e Perspectivas. Movimento, Porto Alegre, v. 15, n. 1, p. 1-25, jan./mar. 2009.

JOMORI, Manuela Mika. Determinantes da escolha alimentar do comensal, durante o almoço num restaurante por peso. 2006, 141 p. Dissertação (Mestrado em Nutrição) Programa de Pós-Graduação em Nutrição, Universidade Federal de Santa Catarina, Florianópolis, 2006

MINAYO, Maria Cecilia de Souza. O desafio do conhecimento: pesquisa qualitativa em saúde. 8. ed. São Paulo: Hucitec, 2004.

PASSOS, Juliana Pereira; FERREIRA, Karla Silva. Caracterização de uma Instituição de Longa Permanência para Idosos e avaliação da qualidade nutricional da dieta oferecida. Alimentos e Nutrição, Araraquara, v. 21, n. 2, p. 241-249, abr./jun. 2010.

PEREIRA, Frances Aparecida llles; CERVATO, Ana Maria. Recomendações Nutricionais. In: PAPALÉO NEITO, Matheus. Gerontologia: a velhice e o envelhecimento em visão globalizada. São Paulo: Atheneu, 2002. p. 248-261.

SANTELLE, Odete; LEFÈVRE, Ana Maria Cavalcanti; CERVATO, Ana Maria. Alimentação institucionalizada e suas representações sociais entre moradores de instituições de longa permanência para idosos em São Paulo, Brasil. Cadernos de Saúde Pública, Rio de Janeiro, v. 23, n. 12, p. 3061-3065, dez. 2007.

SILVA, Edna Lúcia da; CUNHA, Miriam Vieira da. A formação profissional no século XXI: desafios e dilemas. Ciência da Informação, Brasília, v. 31, n. 3, p. 77-82, set./dez. 2002. 